\title{
ESTUDO EPIDEMIOLÓGICO DE ATENDIMENTOS POR CAUSAS EXTERNAS EM UM SERVIÇO PRÉ-HOSPITALAR AÉREO PÚBLICO DO DISTRITO FEDERAL
}

\author{
Wellberson de Souza MACÊDO ${ }^{1}$, Ângela Ferreira BARROS², Alexandre Garcia \\ BARBOSA ${ }^{3}$, Lilyan Paula de Sousa Teixeira LIMA ${ }^{4}$
}

\begin{abstract}
RESUMO: O atendimento às vítimas de causas externas gera um grande impacto para a economia abrangendo a capacidade laborativa, seguridade social e custo dos serviços de saúde, podendo esse impacto também ser percebido nos serviços préhospitalares aéreos. Dessa forma, esse estudo objetiva analisar os atendimentos às vítimas por causas externas por um serviço pré-hospitalar aéreo público do Distrito Federal e verificar fatores associados. Realizou-se um estudo transversal com 595 registros de atendimentos do serviço pré-hospitalar aéreo público do Distrito Federal de 2016 a 2017. Realizou-se modelo simples e múltiplo de regressão logística. Metade dos atendimentos ocorreu às vítimas de causas externas $(53,6 \%)$. Os atendimentos aos casos clínicos $(31,6 \%)$ e transporte $(14,5 \%)$ foram menos frequentes. A maioria dos atendimentos ocorreu no período diurno $(73,6 \%)$. Os atendimentos por causas externas apresentaram maior chance de ocorrer em vítima do sexo masculino (Odds Ratio - OR): 1,99; Intervalo de Confiança (IC) 95\% 1,243,20 ), com idade entre 15 e 29 anos (OR: 12,28; IC 95\% 5,14-29,35), no período noturno (OR: 2,48; IC 95\% 1,33-4,65) e nos finais de semana (OR: 1,61 ; IC 95\% $1,02-2,55)$. O atendimento por causas externas representou importante demanda para o serviço pré-hospitalar aéreo, com maior chance de ocorrerem com homens jovens e acrescentando maior risco ao atendimento por terem maior chance de ocorrerem no período noturno. Isso reforça o forte impacto das causas externas nas perdas humanas, nas incapacidades e nos custos dos serviços de saúde.
\end{abstract}

Palavras-chaves: Resgate aéreo, causas externas, serviços médicos de emergência.

O presente trabalho foi realizado com apoio do Programa de Iniciação Científica da Escola Superior em Ciências da Saúde (ESCS).

\section{INTRODUÇÃO:}

As causas externas anualmente correspondem a mais de cinco milhões de mortes no mundo. Compreendem um conjunto de violência e acidentes evitáveis,

\footnotetext{
1. Acadêmico de Enfermagem. Escola Superior de Ciências da Saúde - ESCS. E-mail: wellber98@hotmail.com.

2. Enfermeira. Doutora. Escola Superior de Ciências da Saúde - ESCS. E-mail: anbarros@yahoo.com.br.

3. Médico. Especialista. Corpo de Bombeiros Militar do Distrito Federal - CBMDF/ Serviço de Atendimento Móvel de Urgência - SAMU. E-mail: drgarciapediatra@gmail.com.

4. Enfermeira. Serviço de Atendimento Móvel de Urgência - SAMU. E-mail: lilyanpls@gmail.com.
} 
assim, incluem-se nesse grupo os diversos tipos de agressões, o suicídio, o acidente automobilístico, o afogamento, a queimadura e a queda. (WHO, 2014).

No contexto brasileiro, as causas externas além de estar entre as dez principais causas de morte no Brasil (MS, 2019), apresentam um aumento que é facilmente percebido nos registros crescentes de internação hospitalar por causas externas nos serviços públicos do Brasil nos últimos anos (MASCARENHAS, 2015), representando um grande impacto para a economia de um país, visto que o tratamento destas é mais oneroso do que por causas naturais (ANDRADE, 2017).

Esse impacto também é percebido pelos serviços pré-hospitalares, que têm como objetivo disponibilizar um atendimento precoce, um transporte adequado e possibilitar uma resposta rápida e resolutiva, sendo o atendimento pré-hospitalar aéreo um diferencial considerando-se a alta densidade populacional das cidades e elevado fluxo rodoviário, em especial para as vítimas de causas externas, tendo em vista que esse tipo de transporte foi associado à maior sobrevida a essas vítimas quando comparado ao transporte terrestre (MICHAELS, 2019).

Nas características dos atendimentos realizados por esse serviço, as causas externas configuram uma demanda frequente (DE LACERDA, 2017), porém o atendimento pré-hospitalar aéreo ainda é pouco aferido e discutido na literatura científica nacional. Dessa forma, esse estudo objetiva analisar os atendimentos às vítimas por causas externas por um serviço pré-hospitalar aéreo público do Distrito Federal e verificar fatores associados a esses atendimentos.

\section{METODOLOGIA:}

Estudo transversal de abordagem quantitativa. A coleta de dados foi realizada no Grupamento de Aviação Operacional do Corpo de Bombeiros Militar do Distrito Federal com dados obtidos das fichas de registros dos atendimentos entre janeiro de 2016 e dezembro de 2017. Foram estudados os aspectos clínicos e sociodemográficos dos atendimentos. A análise dos dados foi realizada no software SPSS v. 20.0 com distribuições em frequência absoluta e medidas de tendência central. Foi realizado um modelo simples e múltiplo de regressão logística. As variáveis que apresentaram $p<0,25$ foram testadas no modelo múltiplo stepwise forward. Permaneceram no modelo múltiplo (ajustado) as variáveis com $p<0,05$.

O presente trabalho foi aprovado pelo Comitê de Ética em Pesquisa do Distrito Federal (FEPECS) pelo parecer consubstanciado $\mathrm{n}^{\circ} 2.836 .265$. 


\section{CONAER

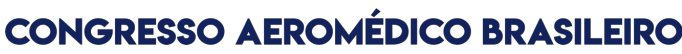

\section{RESULTADOS:}

Foram analisadas 595 ocorrências. A maioria dos atendimentos foi para causas externas $(53,6 \%)$, em seguida para casos clínicos $(31,6 \%)$ e transporte $(14,5 \%)$. Houve maior prevalência de atendimentos no período diurno $(73,6 \%)$ e durante o domingo $(18,5 \%)$, com os atendimentos durante o final de semana representando $34,5 \%$ do total. A aeronave adaptada foi utilizada na maioria das ocorrências (49,9\%) e a especializada foi responsável por 30,6\%.

Em relação às características das vítimas, houve maior prevalência de atendimentos: sexo masculino (67,7\%); faixa etária de 30 a 59 anos $(37,1 \%)$; classificados como críticos $(45,4 \%)$, segundo a escala CIPE.

Quanto à natureza da ocorrência, os acidentes automobilísticos $(59,8 \%)$ foram responsáveis pela maioria das ocorrências por causas externas. Dentre os casos clínicos, as paradas cardiorrespiratórias foram as mais prevalentes (91\%). Entre todas as ocorrências, 30,3\% evoluíram para óbito durante o atendimento.

No modelo múltiplo da regressão logística, comparando os atendimentos por causas externas com os casos clínicos, verificou-se que as vítimas de causas externas apresentaram o dobro de chance de serem do sexo masculino, chance doze vezes maior de ter entre 15 e 29 anos, chance 2,5 maior do atendimento ocorrer no período noturno e $61 \%$ maior chance de ocorrer durante o final de semana. (Tabela 1).

Tabela 1. Variáveis associadas às causas externas no modelo múltiplo de regressão logística

\begin{tabular}{|c|c|c|c|c|c|}
\hline Variáveis & & Categorias & $\mathbf{p}_{\text {(ajustado) }}$ & OR $_{\text {(ajustado) }}$ & IC (95\%) \\
\hline \multirow{2}{*}{ Sexo } & & Feminino & - & 1,00 & - \\
\hline & & Masculino & 0,004 & 1,99 & $1,24-3,20$ \\
\hline \multirow{2}{*}{ Idade } & & $<15$ anos e $>29$ anos & - & 1,00 & - \\
\hline & & Entre 15 e 29 anos & $<0,001$ & 12,28 & $5,14-29,35$ \\
\hline \multirow{2}{*}{$\begin{array}{l}\text { Turno } \\
\text { serviço }\end{array}$} & do & Diurno & - & 1,00 & - \\
\hline & & Noturno & 0,004 & 2,48 & $1,33-4,65$ \\
\hline \multirow{2}{*}{$\begin{array}{l}\text { Dia } \\
\text { semana }\end{array}$} & da & Segunda a sexta & - & 1,00 & - \\
\hline & & Sábado e domingo & 0,040 & 1,61 & $1,02-2,55$ \\
\hline
\end{tabular}




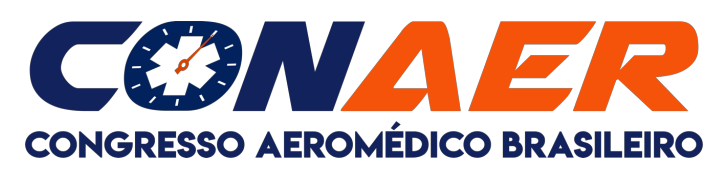

\section{DISCUSSÃO}

A maioria dos atendimentos ocorreu com vítimas do sexo masculino e com o dobro de chances de serem vítimas de causas externas, assim como maior chance de atendimento às vítimas de causas externas no sábado e domingo, semelhante a outros estudos internacionais (ZAIDI, 2019; SOUTO, 2017). Tal fato pode ser decorrente do contexto sociocultural, com forte influência do consumo de álcool e comportamento mais agressivo (LIMA, 2012; MASCARENHAS, 2015),

Os jovens foram os mais atendidos, sendo que pessoas entre 15 e 29 anos tiveram 12 vezes mais chances de serem vítimas de causas externas. Estudos internacionais apontam resultados semelhantes com um impacto notável desse agravo em idade economicamente ativa, além da incidência de incapacidades nessa população, acarretando uma sobrecarga econômica para o país (ANDREW, 2015).

Os atendimentos às vítimas de causas externas apresentaram o dobro de chances de ocorrer no período noturno. Devido à maior risco e complexidade, os atendimentos nesse período devem ser realizados com pouso em locais previamente estabelecidos gerando uma cultura de segurança que previne possíveis erros (MACDONALD, 2008).

Apesar da limitação desse estudo no acesso à dados secundários os presentes resultados agregam aos serviços de saúde e sociedade a perspectiva de atendimento pré-hospitalar capaz de aumentar a sobrevida de pacientes vítimas de causas externas, conforme verificado em estudo prévio (MICHAELS, 2019).

\section{CONSIDERAÇÕES FINAIS}

O presente estudo constatou que as causas externas representaram um pouco mais da metade dos atendimentos do serviço pré-hospitalar aéreo público do Distrito Federal nos anos de 2016 e 2017 estando associadas ao sexo masculino, idade entre 15 e 29 anos, período noturno e finais de semana.

Além disso, essa pesquisa divulga informações acerca da natureza especializada do tipo de atendimento realizado, incrementa o acervo de pesquisas com a temática do atendimento pré-hospitalar aéreo brasileiro, além de agregar informações que podem ser utilizadas para melhoria das normas, rotinas e registros dos atendimentos, assim como, fomentar perspectivas de aprimoramento do serviço. 


\section{REFERÊNCIAS}

ANDRADE, S. S. C. A; JORGE, M. H. P. M. Internações hospitalares por lesões decorrentes de acidente de transporte terrestre no Brasil, 2013: permanência e gastos. Epidemiol. Serv. Saude. v. 26, n. 1. p. 31-8. 2017.

ANDREW E., et al. Characteristics of Patients Transported by a Paramedic-staffed Helicopter Emergency Medical Service in Victoria, Australia. Prehosp Emerg Care. v. 19, n. 3. p. 416-24. 2015.

DE LACERDA, L. S.; ARAÚJO, E. R.; NETA, F. L. A. Transporte aeromédico no estado do Piauí: perfil das ocorrências. Rev Pre Infec e Saúde. v. 3, n. 2. p. 20-6. 2017.

LIMA, A. L. A. et al. Mortalidade por causas externas nos municípios de Arapiraca (AL) e Mossoró (RN) - 1999-2008. Revista Baiana de Saúde Pública. v. 36, n. 1. p. 134-47. 2012.

MACDONALD, R. D,; BANKS, B. A.; MORRISON, M. Epidemiology of adverse events in air medical transport. Acad Emerg Med. v. 15, n. 10. p. 923-31. 2008.

MASCARENHAS, M. D. M.; BARROS M. B. A. Evolução das internações hospitalares por causas externas no sistema público de saúde - Brasil, 2002 a 2011. Epidemiol. Serv. Saúde. v. 24, n. 1. p. 19-29. 2015.

MICHAELS, D. et al. Helicopter versus ground ambulance: review of national database for outcomes in survival in transferred trauma patients in the USA. Trauma Surg Acute Care Open. v. 4, n. 1. p. 1-3. 2019.

MINISTÉRIO DA SAÚDE. Principais causas específicas de morte no Brasil e regiões, e mortalidade prematura por DCNT, 2005 a 2016. In: Saúde Brasil 2018 uma análise de situação de saúde e das doenças e agravos crônicos: desafios e perspectivas. Brasília: Ministério da Saúde. p. 75-92. 2019.

SOUTO R. M. C. V., et al. Perfil epidemiológico do atendimento por violência nos serviços públicos de urgência e emergência em capitais brasileiras, Viva 2014. Cien Saude Colet. v. 22, n. 9. p. 2811-2823. 2017.

WORLD HEALTH ORGANIZATION. Injuries and violence: the facts 2014. Report. Geneva: WHO; 2014.

ZAIDI A. A. et al. The burden of trauma at a district hospital in the Western Cape Province of South Africa. Afr J Emerg Med. v. 9. p. 14-20. 2019. 